\title{
Antibody response induced by the boost overdose during COVID-19 heterologous prime-boost vaccination strategy: $A$ four-case study
}

Francisco Raposo ( $\square$ fraposo@ig.csic.es )

CSIC-Instituto de la Grasa

Giuseppe Lippi

University of Verona

\section{Research Article}

Keywords: Antibody response monitoring, Anti-SARS-CoV-2 RBD Ig G antibody, BNT162b2 vaccine, COVID-19, SARS-CoV-2 virus, Vaccination, Vaccine overdose

Posted Date: September 9th, 2021

DOl: https://doi.org/10.21203/rs.3.rs-885163/v1

License: (c) (i) This work is licensed under a Creative Commons Attribution 4.0 International License.

Read Full License 


\section{Abstract \\ Objectives}

The main purpose of this study was to evaluate the anti-SARS-CoV-2 RBD Ig G antibody response in BNT162b2 vaccine recipients who erroneously received vaccine overdose.

\section{Methods}

Measurement of antibody levels at different time-points was performed to define the dynamics of immunization after a wrongly vaccination schedule. Three recipients had no previous evidence of infection and received the first shot of Oxford/AstraZeneca before the Pfizer/BioNTech vaccine. Another patient, formerly infected by SARS-CoV-2, received one shot of BNT162b2 vaccine.

\section{Results}

At day 6 after the second vaccine dose the serum increase of anti-SARS-CoV-2 RBD Ig G antibodies was analogous for the three SARS-CoV-2 naïve recipients. At 14 days the antibody level increased and reached a peak, though displaying a different pattern among the three recipients. At 21 days the serum antibody level started to decrease from its maximum value. The data for the previously infected recipient were in agreement with values found in COVID-19 positive receivers. Thus, after the single prime-dose of vaccine, the elicited antibody response was similar to prime-boost vaccination in naïve recipients.

\section{Conclusions}

This study confirms the efficiency of the BNT162b vaccine in eliciting a sustained antibody response as heterologous boost-vaccine in previously Oxford/AstraZeneca vaccinated recipients, as well as, primevaccine in COVID-19 infected receivers. Importantly, the humoral immunity response of recipients was not proportional to the vaccine overdose. Nonetheless, we cannot portray a univocal effect of vaccine overdose concerning anti-SARS-CoV-2 antibody response because the values found especially in the three SARS-CoV-2 naïve subjects were highly heterogeneous.

\section{Introduction}

Coronaviruses are a large family of viruses which assume the prefix "corona," from the many crown-like spikes on their surface. The novel SARS-CoV-2 coronavirus which sustains the current pandemic outbreak appears as a completely new pathogen, sharing modest similarities with SARS-CoV- 1 and MERS-CoV. It was firstly identified in Wuhan China in December 2019 and since then it has now spread worldwide. SARS-CoV-2 contains four structural proteins distributed in the form of one internal protein (nucleocapsid) and three external proteins (envelope, membrane, and spike). The nucleocapsid protein is 
inside the virus, associated with viral RNA, whilst the spike protein contains the receptor-binding domain (RBD) to natural receptors on host cells surfaces, mainly angiotensin-converting enzyme 2 (ACE 2) [1].

Due to the ongoing COVID-19 pandemic, vaccination continues to be critically important because it is regarded as safe and effective means to prevent disease and reduce virulence [2,3]. The current COVID19 vaccines are not specifically designed to avoid contracting viral infection since the main target of vaccination now is focused on eliciting an immune response against the virus and thus lowering its pathogenicity (i.e. protecting against serious illness or death) $[4,5]$. COVID-19 vaccines have been developed following equivalent legal requirements for efficacy and safety as for all other vaccines. In the European Union, before being made commercially available, any COVID-19 vaccine candidate needs to meet the severe requirements and evidence thresholds of the European Medicines Agency (EMA) [6]. What is different in such case is that development and approval have been much faster, due to the publichealth emergency caused by this new coronavirus. COVID-19 vaccination campaign across the European Union started on 27 December 2020, and it is still in progress. The European Commission has so far given the provisional marketing approval for 4 vaccines (Pfizer/BioNTech, Moderna, Oxford/AstraZeneca and Janssen Pharmaceuticals) [7].

In order to boost the vaccination rate some different strategies have been proposed such as splitting the doses, delaying the second dose, heterologous vaccination, delay of vaccination in seropositive recipients [8]. In addition, a practical problem with some types of vaccines is the multi-vial dose format and the need of preparation before administration, whereby its contribution to vaccine wastage (sum of vaccines discarded, damaged and lost) must be reduced. Finally, one more controversial subject is the anti-SARSCoV-2 serological monitoring of COVID-19 vaccinated population [9].

For vaccines that require two injections, healthcare organizations have typically recommended the second shot to be the same as the former. Following safety issues, mostly related to cases of atypical venous thrombosis, some European countries have decided to stop the use of the adenovirus-based Oxford/AstraZeneca vaccine. Consequently, millions of people were unable to receive a second dose of such vaccine, remaining only partially vaccinated. To resolve this matter, mix-and-match vaccine studies have been planned aimed to investigate the safety and immune response in people receiving two different types of COVID-19 vaccine [10-11]. Some preliminary studies carried out in different countries suggest that combining different vaccines induces potent immune response [12-15], but some safety concerns remain [16].

The BNT162b2 mRNA COVID-19 vaccine has been designed to be given in two $30 \mu \mathrm{g}$ doses, 21 days apart [17]. Millions of doses have been now administered around the world, but in some cases vaccine overdose has occurred, mostly due to human errors. A summary of the cases reported worldwide is as follows: Israel-Jerusalem (3); Germany-Stralsund (8); Singapore-Singapore (1); Australia-Brisbane (2); USA-Ford Madison (77); Italy-Massa (1); Spain-Seville (8). In addition, the company informed in the vaccine brochure that during a clinical trial 52 participants received an erroneous dilution equivalent to a nearly double dose of vaccine $(58 \mu \mathrm{g})$ [17]. In these cases, the mistake was due to the use of "multi-dose" 
vials [18] which are useful in a pandemic situation because they allow cheaper and more efficient distribution. However, multi-dose vaccines, mainly when the vaccine needs to be reconstituted before injection, are more prone to administration errors. Specifically, each single vial of the Pfizer/BioNTech vaccine contains multiple doses (between five and six, in general). The active component of the vaccine $(0.45 \mathrm{~mL})$ must be diluted using $0.9 \%$ sodium chloride $(1.8 \mathrm{~mL})$. As a consequence, dosing errors may be caused by omitting the mandatory dilution by healthcare operators, so that a concentrated (higher dosage) product could be mistakenly injected.

Therefore, the main purpose of this study was to report the anti-SARS-CoV-2 RBD immunoglobulin class $\mathrm{G}(\mathrm{lg} \mathrm{G})$ antibody response in recipients of erroneous Pfizer/BioNTech vaccination schedule (overdosage)

\section{Material \& Methods}

\subsection{Ethical statement}

All participants provided written informed consent to publish their analytical antibody values. The study complied with all the relevant national regulations, institutional policies and in accordance the tenets of the Helsinki Declaration regarding ethical conduct of research involving human subjects.

\subsection{Vaccine recipients}

This four-case study was based on two males and two females, aged between 42-53 years. All this four vaccine overdose recipients (VORs) received $0.3 \mathrm{~mL}$ of the undiluted multi-dose vial, that means a vaccine dose increased by 5 -fold $(150 \mu \mathrm{g})$. Three recipients (VOR-1, 2 and 3 ) had no previous evidence of infection and received the first shot of Oxford/AstraZeneca vaccine 14.5 weeks before undergoing a second vaccination with Pfizer/BioNTech vaccine. The remaining subject (VOR-4) who had been previously infected by SARS-CoV-2 only received a single dose of Pfizer/BioNTech vaccine. Venous blood was take at three different time points, i.e. $6\left(T_{6}\right), 14\left(T_{14}\right)$ and 21 days $\left(T_{21}\right)$ after the administration of the Pfizer/BioNTech vaccine.

The serum basal value $\left(T_{0}\right)$ was arbitrarily set at $57 \mathrm{BAU} / \mathrm{mL}$, in accordance with values found in previous studies $[12,19]$.

\subsection{SARS-CoV-2 RBD Ig G antibody measurement}

The concentration of binding antibody can be found frequently in COVID-19 vaccine immunity studies using different methodologies with diverse measurements units [20].

In this report, Abbott SARS-CoV-2 Ig G Quant ${ }^{\circledR}$ assay was used as methodology for the quantitative assessment of anti-SARS-CoV-2 Ig $\mathrm{G}$ antibodies using the Architect platform (Abbott Laboratories Abbott Park, IL, USA). This is an automated two-step chemiluminiscent microparticle immunoassay (CMIA) designed to detect specific lg $\mathrm{G}$ antibodies to the RBD of S1 subunit of SARS-CoV-2 spike protein. All samples were processed by in accordance with manufacturers' instructions including the use of 
calibrators and controls. Results of this assay are arbitrary expressed as $\mathrm{AU} / \mathrm{mL}$. The limit of quantitation is $21 \mathrm{AU} / \mathrm{mL}$, the analytical measuring interval ranges from $21-40000 \mathrm{AU} / \mathrm{mL}$ (can be extended to 80000 $\mathrm{AU} / \mathrm{mL}$ with $1: 2$ diluted samples). The intermediate precision ranges between 3-5\% CV whilst the trueness, as \% apparent recovery, is $\pm 5 \%$. Samples are considered positive when the value is $\geq 50 \mathrm{AU} / \mathrm{mL}$ [21]. After the first WHO international standard for anti-SARS-CoV-2 immunoglobulin has been released by the National Institute for Biological Standards and Control at the end of 2020 (NIBSC code 20/136) [22] the results for anti-SARS-CoV-2 antibodies testing shall be expressed as binding antibody units per milliliter (i.e., BAU/mL) [23]. For the assay used in this study, the conversion we used for reporting values in binding antibody units was as follows: $\mathrm{BAU} / \mathrm{mL}=\mathrm{AU} / \mathrm{mL}$ * $0.143[20]$

\section{Results}

\subsection{Humoral response}

The results of the anti-SARS-CoV-2 RBD IG G antibodies levels measured in the four VORs at the three time points are summarized in table 1 . Since the mean reason that prompted this study was the consequence of a human error, the basal serum values of anti-SARS-CoV-2 RBD Ig G antibodies before the administration of Pfizer/BioNTech vaccine are unavailable.

After Pfizer/BioNTech vaccine administration, a similar increase of serum anti-SARS-CoV-2 RBD Ig G antibodies levels was recorded at the first time point $\left(T_{6}\right)$, with an approximate increase in the $T_{6} / T_{0}$ ratio of around 12 -fold. At this time the concentration value for the one shot vaccine recipient (VOR-4) was nearly double compared to prime-boost vaccine recipients (VOR-1, 2 and 3 ). During the following week after Pfizer/BioNTech vaccine administration $\left(T_{14}\right)$, the antibody level increased and reached a peak, but following a quite different pattern among the three VORs. At this time, the $T_{14} / T_{6}$ ratio values were 1.6, 3.8, 9.5 and 4.0 for VOR-1,2,3 and 4, respectively, whilst the $T_{14} / T_{0}$ ratio values were 22,46 and 114 for VOR-1, 2 and 3, respectively. Finally, the antibody level decreased from the maximum values during the third week after the Pfizer/BioNTech vaccine administration $\left(T_{21}\right)$. At the third time point, the $T_{21} / T_{14}$ ratio values were on average $\otimes 0.78$. Similarly, the $T_{21} / T_{0}$ ratio values were 18,34 and 89 for VOR-1, 2 and 3, respectively. At this time point, the result for VOR-4 was similar to the highest value obtained for VOR-3, and considerably higher that the values recorded in VOR-1 and VOR-2. For VOR-4, the value was in keeping to those previous found in positive COVID-19 recipients in whom the single primedose of BNT162b2 vaccine induced an antibody response similar to a full prime-boost dose in naïve recipients [24, 25].

\subsection{Side effects}

No major systemic side effects could be recorded after administration of vaccine overdose in VORs recipients. The most commonly reported systemic events were headache and general malaise. Also, in some cases, fever and tiredness were described. Additionally, mild/moderate pain at the injection site, 
lasting between 72-96 h, was the most frequently described local reaction in VORs after vaccine administration.

\section{Discussion}

Despite there are some drawbacks in the present report such as the limited number of subjects studied, the short timeframe after immunization and the lack of information on virus neutralization or cellular immune response, we have provided interesting evidence on the serological response to COVID-19 vaccine overdosage. The specific nature of VORs is an important novelty from the scientific perspective, that prompted us to describe these cases. Basically, the VORs' immugenicity found in our study was compared with experimental values relating to COVID-19 vaccination studies available from the current scientific. It is important to note that research groups working on this field described a range of immunoassays with different readouts to measure antibody binding responses after immunization, which critically make difficulties regarding to the comparisons of datasets [20].

As concerns previously published data, the Pfizer/BioNTech consortium has carried out different trials for selecting type and dose of mRNA-based COVID-19 vaccine. In particular, Vogel and co-workers reported the preclinical development of two m-RNA based vaccine candidates (BNT162b1 versus BNT162b2) [26]. Non-human primates (NHP) were intramuscularly injected with two different dose levels of vaccine (30 $\mu \mathrm{g}$ and $100 \mu \mathrm{g}$ ) at days 0 and 21. The anti-SARS-CoV-2 RBD Ig G were measured with a fluorescent detection assay. Antibodies were detected by day 14 after the first dose, and the level increased until to reach a peak the day 28 ( 7 days after second vaccine dose). At day 28 , the levels were 23781 and $26170 \mathrm{U} / \mathrm{mL}$ for 30 and $100 \mu \mathrm{g}$ dose level, respectively. These values declined to 2846 and $5442 \mathrm{U} / \mathrm{mL}$ by day 56 (i.e., 35 days after second vaccine dose), respectively. Two important findings emerged from this study. Firstly, the antibody values in 38 SARS-CoV-2 convalescent human sera was $602 \mathrm{U} / \mathrm{mL}$, which is lower compared to the values of immunized NHP after only the first vaccine dose of $30 \mu \mathrm{g}$ (i.e., $1488 \mathrm{U} / \mathrm{mL}$ ). Secondly, compared to the $30 \mu \mathrm{g}$ vaccine dose, the increase of anti-SARS-Cov-2 RBD Ig G increment was only $10 \%$ for the $100 \mu \mathrm{g}$ dose at the maximum concentration level (day 28), and ranged between 25-33\% throughout the 21-42 days period, thus confirming that a 3-fold higher vaccine dosage does not elicit a similar considerable increase of humoral immunity. On the other hand, Walsh and co-workers [27] and Sahin and co-workers [28] carried out further phase 1 trials of BNT162b2 vaccine. Unfortunately, considering the dose levels of such studies it was not possible to be comparatively evaluate the antibody immunity response regarding to the vaccine overdose schedule.

In another study using recipients only partially protected with a first dose of Oxford/AstraZeneca vaccine, Borobia and co-workers randomly assigned them between two groups, where the "intervention" group received a second dose of BNT162b2 vaccine while the "control" group was maintained with the previously injected first dose of vaccine [12]. Humoral response monitored with Roche-Elecsys ${ }^{\circledR}$ antiSARS-CoV-2 RBD Ig G [29] displayed a robust immune response regarding to the heterologous vaccination schedule. At 7 days the value was $4354 \mathrm{BAU} / \mathrm{mL}$ in the intervention group versus $90 \mathrm{BAU} / \mathrm{mL}$ in the control group. For the following time point of 14 days, the values increased until 7757 versus 100 
$\mathrm{BAU} / \mathrm{mL}$, respectively. Unfortunately, the values cannot be comparatively evaluated because data obtained using Abbot-Quant ${ }^{\circledR}$ methodology, although expressed as $\mathrm{BAU} / \mathrm{mL}$, are in general lower than reported by Roche-Elecsys ${ }^{\circledR}[20]$. For example, the maximum reported value for VORs at 14 days was $6500 \mathrm{BAU} / \mathrm{mL}$, which it is lower than 95\% C.I (7156-8161) of CombiVacs study results.

Published information on immunogenicity after full-dose vaccine administration is still occasional. However, some experimental results from the literature as regards to the immune response after the second dose of BNT162b2 vaccine in a homologous schedule were evaluated [20,30-36]. The required conditions for the comparative study of VORs data were identical methodology (Abbott-Quant ${ }^{\circledR}$ ) and exact time point for blood sampling ( \pm 1 day). The values for specific antibodies concentration are summarized in table 2 . There are a few studies reporting the baseline value before receiving the second vaccine dose, which values ranged between 114-239 BAU/mL. At 7 and 14 days the values for naïve recipients ranged between 2136-2967 and 2203-2796 BAU/mL, respectively. At this time points the values can be considered as lower, similar and higher for VOR-1, 2 and 3, respectively. In addition, the kinetic in humoral response regarding to anti-SARS-CoV-2 RBD Ig G was different when compared to NHP recipients. In the case of VORs, the maximum values were achieved during the second instead of the first week after the boost vaccine administration.

\section{Conclusions}

All the VORs included in our report developed a significant humoral immunity between 6-21 days after vaccination. In spite of the overdosage, this study hence confirms the double efficiency of the BNT162b2 mRNA COVID-19 vaccine concerning to generation of a SARS-CoV-2 RBD Ig G antibody response induced as heterologous boost-vaccine in previously Oxford/Astra-Zeneca vaccinated recipients. But also provides evidence as prime-vaccine in COVID-19 infected receivers. The humoral immunity responses for VORs were very heterogeneous considering that a similar vaccine overdose has been injected. The common evidence is limited to the fact that all the VORs achieved the maximum level of anti-SARS-CoV-2 $R B D$ Ig $G$ values at the second week after vaccination, and then decreased. In spite of this similar trend, significant differences in concentration levels among recipients of this study does not permit to report an unequivocal effect of vaccine overdose. This is due to all theoretically possible individual results for antibody responses such as attenuated, averaged and increased levels were stated.

\section{Declarations}

Competing interests: The authors declare no competing interests.

\section{References}

1. Zhang H, Penninger JM, Li Y, Zhong N, Slutsky AS, Angiotensin-converting enzyme 2 (ACE2) as a SARS-CoV-2 receptor: molecular mechanisms and potential therapeutic target, Intensive Care Med. 46 (2020) 586-590. https://doi.org/10.1007/s00134-020-05985-9. 
2. Haas EJ, Angulo FJ, McLaughlin JM, Anis E, Singer SR, Khan F, et al., Impact and effectiveness of mRNA BNT162b2 vaccine against SARS-CoV-2 infections and COVID-19 cases, hospitalizations, and deaths following a nationwide vaccination campaign in Israel: an observational study using national surveillance data, The Lancet 397 (2021) 1819

3. Koch T, Mellinghoff SC, Shamsrizi P, Addo MM, Dahle C, Correlates of vaccine-induced protection against SARS-CoV-2, Vaccines 9 (2021) 238 https://doi.org/10.3390/vaccines 9030238

4. Klasse PJ, Nixon DF, Moore JP, Immunogenicity of clinically relevant SARS-CoV-2 vaccines in nonhuman primates and humans, Sci. Adv. 7 (2021) https://doi.org/10.3390/vaccines9030238

5. Lombardi A, Bozzi G, Ungaro R, Villa S, Castelli V, Mangioni D, Muscatello A, et al., Mini-review immunological consequences of immunization with COVID-19 mRNA vaccines: preliminary results, Frontiers in immunology (2021) 12 https://doi.org/10.3389/fimmu.2021.657711

6. European Medicines Agency. Human regulatory: COVID-19 updates. https://www.ema.europa.eu/en/human-regulatory/overview/public-health-threats/coronavirusdisease-covid-19/covid-19-latest-updates. Accessed July, 2021

7. European Commission. Safe COVID-19 vaccines for Europeans. https://ec.europa.eu/info/live-worktravel-eu/coronavirus-response/safe-covid-19-vaccines-europeans_en. Accessed July, 2021

8. Brüssow H, COVID-19: vaccination problems, Environ. Microbiol. 23 (2021) 2878-2890. https://doi.org/10.1111/1462-2920.15549.

9. Lippi G, Henry BM, Plebani M, Anti-SARS-CoV-2 antibodies testing in recipients of COVID-19 vaccination: Why, when, and how?, Diagnostics 11 (2021) 1-10. https://doi.org/10.3390/diagnostics11060941

10. Callaway E, Mixing COVID vaccines triggers potent immune response, Nature. 593 (2021) 491.

11. Lewis $D$, The case is growing for mix-and-match COVID vaccines, Nature 595 (2021) 344-345. https://doi.org/10.1038/d41586-021-01805-2

12. Borobia AM, Carcas AJ, Pérez-Olmeda M, Castaño L, Bertran MJ, García-Pérez J, et al., Immunogenicity and reactogenicity of BNT162b2 booster in ChAdOx1-S-primed participants (CombiVacS): a multicentre, open-label, randomised, controlled, phase 2 trial, Lancet. 6736 (2021) 110. https://doi.org/10.1016/s0140-6736(21)01420-3.

13. Hillus D, Schwarz T, Tober-Lau P, Hastor $H$, Thibeault C, Kasper $S$ et al., Safety, reactogenicity, and immunogenicity of homologous and heterologous prime-boost immunisation with ChAdOx1-nCoV19 and BNT162b2: a prospective cohort study, MedRxiv. (2021) 2021.05.19.21257334. https://www.medrxiv.org/content/10.1101/2021.05.19.21257334v2.

14. Schmidt T, Klemis V, Schub D, Mihm J, Hielscher F, Abu-Omar A et al., Immunogenecity and reactogenicity of a heterolougous COVID-19 prime-boost vaccination compared with homologous vaccine regimens, MedRxiv (2021) 2021.06.13.21258859 https://www.medrxiv.org/content/10.1101/2021.06.13.21258859.

15. Liu X, Shaw RH, Stuart ASV, Greenland M, Dinesh T, Provstgaard-Morys et al., Safety and immunogenecity report from the Com-Cov study-A single-blind randomized non-inferiority trial 
comparing heterologous and homologous prime-boost schedules with an adenoviral vectored and mRNA COVID-19 vaccine, SSRRN (2021)

16. Shaw RH, Stuart A, Greenland M, Liu X, Van-Tam JSN, Snape MD, Heterologous prime-boost COVID19 vaccination: initial reactogenicity data, Lancet. 397 (2021) 2043-2046.

https://doi.org/10.1016/S0140-6736(21)01115-6.

17. ComiRNAty leaflet. Summary of product characteristics. Available at:

https://www.ema.europa.eu/en/documents/product-information/comirnaty-epar-productinformation_en.pdf

18. Paparella S, The risk associated with the use of multidose vials, J Emerg Nurs 32 (2006) 428-430

19. Eyre DW, Lumley SF, Wei J, Cox S, James T, Justice A, et al. Quantitative SARS-CoV-2 anti-spike responses to Pfizer-BioNTech and Oxford-AstraZeneca vaccines by previous infection status. Clin Microbiol Infect 2021. https://doi.org/10.1016/j.cmi.2021.05.041

20. Perkmann T, Perkmann-Nagele N, Koller T, Mucher P, Radakovics A, Marculescu R et al., Anti-Spike protein assays to determine post-vaccination antibody levels: a head-to-head comparison of five quantitative assays, MedRxiv. (2021) 2021.03.05.21252977.http://medrxiv.org/content/early/2021/03/08/2021.03.05.21252977.abstract

21. Abbott Core Laboratory: SARS-CoV-2 Immunoassays: Advancing diagnostics of COVID-19. Available at: https://www.corelaboratory.abbott/int/en/offerings/segments/infectious-disease/sars-cov-2. Accessed July, 2021.

22. First WHO International Standard for anti-SARS-CoV-2 immunoglobulin. 2021. https://www.who.int/groups/expertcommittee-on-biological-standardization (accessed March 8, 2021).

23. Kristiansen PA, Page M, Bernasconi V, Mattiuzzo G, Dull P, Makar K, et al., WHO International Standard for anti-SARS-CoV-2 immunoglobulin. The Lancet. 397 (2021) 1347-8. https://doi.org/10.1016/S0140-6736(21)00679-6

24. Callegaro A, Borleri D, Farina C, Napolitano G, Valenti D, Rizzi M, et al. Antibody response to SARSCoV-2 vaccination is extremely vivacious in subjects with previous SARS-CoV-2 infection. J Med Virol. 2021;93(7):4612-5. https://doi.org/10.1002/jmv.26982

25. Ebinger JE, Fert-Bober J, Printsev I, Wu M, Sun N, Prostko JC, et al. Antibody responses to the BNT162b2 mRNA vaccine in individuals previously infected with SARS-CoV-2. Nat Med [Internet]. 2021;27(6):981-4. http://dx.doi.org/10.1038/s41591-021-01325-6

26. Vogel AB, Kanevsky I, Che Y, Swanson KA, Muik A, Vormehr M et al., BNT162b vaccines protect rhesus macaques from SARS-CoV-2, Nature. 592 (2021) 283-289. https://doi.org/10.1038/s41586021-03275-y.

27. Walsh EE, Frenck RW, Falsey AR, Kitchin N, Absalon J, Gurtman A et al., Safety and immunogenicity of two RNA-Based Covid-19 vaccine candidates, N. Engl. J. Med. 383 (2020) 2439-2450. https://doi.org/10.1056/nejmoa2027906. 
28. Sahin U, Muik A, Vogler I, Derhovanessian E, Kranz LM, Vormehr M et al., BNT162b2 vaccine induces neutralizing antibodies and poly-specific T cells in humans, Nature. (2021). https://doi.org/10.1038/s41586-021-03653-6

29. Elecsys ${ }^{\circledR}$. Anti-SARS-CoV-2 S. Immunoassay for the quantitative determination of antibodies to the SARS-COV-2 spike protein. https://diagnostics.roche.com/gb/en/products/params/elecsys-anti-sarscov-2-s.html

30. Bradley et al. Anti-SARS-CoV-2 antibody levels are concordant across multiple platforms but are not fully predictive of sterilizing immunity. $2021 \mathrm{https} / / /$ doi.org/10.1101/2021.04.26.21256118

31. Kanji JN, Bailey A, Fenton J, Ling SH, Rivera R, Plitt S, et al. Detection of SARS-CoV-2 antibodies formed in response to the BNT162b2 and mRNA-1237 mRNA vaccine by commercial antibody tests. medRxiv [Internet]. 2021;2021.03.30.21254604. https://www.medrxiv.org/content/10.1101/2021.03.30.21254604v1

32. Kontopoulo K, Ainatzoglou A, Nakas CT, Ifantidou A, Gkounti G, Antoniadou E. Second dose of the BNT162b2 mRNA vaccine:the value of timely administration. https://ssrn.com/abstract $=3800040$

33. Ontañon J, Blas J, de Cabo C, Santos C, Ruiz-Escribano E, García A, Influence of past infection with SARS-CoV-2 on the response to the BioTech/ Pfizer BNT162b2 mRNA vaccine in health care workers: kinetics and durability of the humoral response. medRxiv Prepr. 2021;1-10. https://doi.org/10.1101/2021.05.25.21257788

34. Velasco M, Galán MI, Casas ML, Pérez-Fernández E. Impact of previous COVID-19 on immune response after a single dose of BNT162b2 SARS-CoV-2 vaccine. medRxiv Prepr. 2021; https://doi.org/10.1101/2021.03.08.21253065

35. Zee JS, Lai KT, Ho MK, Leung AC, Chan QW, Ma ES, et al. Serological response to mRNA and inactivated COVID-19 vaccine in healthcare workers in Hong Kong: preliminary results. Hong Kong Med J. 2021;1-2. https://doi.org/10.12809/hkmj219605

36. D. Zipeto, L.D. Carbonare, M.T. Valenti, Z. Bisoffi, C. Piubelli, M. Pizzato, S. Accordini, S. Mariotto, S. Ferrari, A. Minoia, J. Bertacco, V.L. Vigni, G. Dorelli, E. Crisafulli, D. Alberti, L. Masin, S. Longoni, L. Lopalco, A. Beretta, N. Tiberti, Antibody response to BTN162b2 mRNA vaccination in naïve versus SARS-CoV-2 infected subjects with and without waning immunity, 2021. https://doi.org/10.21203/rs.3.rs-440410/v1.

\section{Tables}

Table 1.Concentration of anti-SARS-CoV-2 RBD Ig G (BAU/mL) at different time points 


\begin{tabular}{|llll|}
\hline Recipient number & \multicolumn{4}{l}{ Time elapsed from Pfizer/BioNTech vaccine administration } \\
\hline & $\mathrm{T}_{6}=6$ days & $\mathrm{T}_{14}=14$ days & $\mathrm{T}_{21}=21$ days \\
\hline VOR-1 & $780(39)$ & $1250(63)$ & $1040(52)$ \\
\hline VOR-2 & $690(34)$ & $2600(132)$ & $1930(96)$ \\
\hline VOR-3 & $680(34)$ & $6500(324)$ & $5000(253)$ \\
\hline VOR-4 & $1370(68)$ & $5500(274)$ & $4300(214)$ \\
\hline
\end{tabular}

Table 2. Concentration of anti-SARS-CoV-2 RBD $\lg \mathrm{G}(\mathrm{BAU} / \mathrm{mL})$ from literature

\begin{tabular}{|c|c|c|c|c|c|}
\hline \multirow[t]{2}{*}{ Reference } & \multicolumn{5}{|c|}{$\begin{array}{l}\text { Time elapsed from Pfizer/BioNTech } \\
\text { second vaccine dose administration (days) }\end{array}$} \\
\hline & 0 & 7 & 14 & 21 & 28 \\
\hline Bradley et al. [30] & 114 & & & 2419 & \\
\hline Kanji et al. [31] & 169 & & & & \\
\hline Kontopoulou et al. [32] & & & 2136 & & \\
\hline Ontañon et al. [33] & 176 & 2892 & 2967 & 2203 & \\
\hline Perkmann et al. [20] & 214 & & & & \\
\hline Velasco et al. [34]-naive & 146 & & & & \\
\hline previously infected & 3184 & & & & \\
\hline Zee et al. [35] & 231 & & & & 1655 \\
\hline Zipetto et al. [36]-naive & 239 & & & 2796 & \\
\hline previously infected & 2879 & & & & \\
\hline
\end{tabular}

